\title{
Comparação entre duas escalas de segurança alimentar
}

\author{
A comparison between two food security scales
}

Leonardo Pozza dos Santos ${ }^{1}$

Mariana Gamino da Costa ${ }^{2}$ Janaína Vieira dos Santos ${ }^{1}$

Ivana Loraine Lindemann ${ }^{2}$

Denise Petrucci Gigante ${ }^{1}$

${ }^{1}$ Programa de PósGraduação em Epidemiologia, Universidade Federal de Pelotas, Brasil. leonardo_pozza@ yahoo.com.br

${ }^{2}$ Faculdade de Nutrição, Universidade Federal de Pelotas, Brasil.
Abstract The scope of this study was to evaluate the influence of the social determinants and the provision of primary care services in relation to the use of emergency dental care services in a medium-sized municipality. Data recorded for the 57,231 users of emergency care between 2007 and 2009, in accordance with age, gender, date and period of dental care, social exclusion indices of the suburb in which they live and the existence of a benchmark oral health team, were used to perform the analysis. Of the total population, 5.24\% on average per year used the service during the period under scrutiny, with the 20-49 year age group (63.84\%) showing the highest demand and equality between genders for such care. Surgical procedures (54.90\%) were the most prevalent with an increasing trend for restorative procedures $(62,8 \%)$. Users living in areas of greater social exclusion were 4.15 times more likely to seek dental care $(p<0.05)$. No statistically significant association was found between gender or the existence of an oral health team of the suburb in which they live and the demand for emergency dental care. In conclusion, there was greater recourse to emergency municipal dental care by individuals living in vulnerable areas, proving the importance of such care in diminishing oral health inequality.

Key words Oral health, Emergencies, Epidemiology
Resumo Este artigo tem por objetivo comparar a versão curta da escala de segurança alimentar, recomendada pela FAO, e a Escala Brasileira de Insegurança Alimentar (EBIA). Foi utilizado um questionário com as duas escalas, além de informações demográficas e socioeconômicas. Os indices de sensibilidade, especificidade, valores preditivos positivo e negativo e acurácia foram obtidos pela comparação dos valores entre as escalas. Com o intuito de avaliar a concordância entre os dois instrumentos, aplicou-se o teste Kappa. Das 230 famílias estudadas, mais da metade convivia com algum nível de insegurança alimentar, segundo a EBIA, e apenas 1/4 da amostra vivia nessa situação, de acordo com a versão curta. A comparação entre as escalas mostra concordância moderada tanto para analisar insegurança alimentar como um todo, quanto para analisar uma possível situação de fome, mesmo alterando o ponto de corte de ambas. Na análise dos parâmetros de validade, constatou-se que a versão curta apresenta baixa sensibilidade e alta especificidade em relação à EBIA. A versão curta apresenta baixa concordância quando comparada à EBIA. Logo, faz-se necessário a validação de um instrumento menor em relação à EBIA, de rápida e fácil aplicação.

Palavras-chave Segurança alimentar e nutricional, Estudos de Validação, Escalas 


\section{Introdução}

A segurança alimentar e nutricional consiste na realização do direito de todos ao acesso regular e permanente a alimentos de qualidade, em quantidade suficiente. Além disso, não deve comprometer o acesso a outras necessidades essenciais, tendo como base práticas alimentares promotoras de saúde que respeitem a diversidade cultural e que sejam ambiental, cultural, econômica e socialmente sustentáveis ${ }^{1}$.

Na década de 1990, nos Estados Unidos, foi desenvolvido o primeiro instrumento capaz de dar uma dimensão à insegurança alimentar. Foi criada uma escala de 18 itens que possibilita abranger todos os níveis de severidade da insegurança alimentar observados nos domicílios com e sem crianças. Tal escala é a recomendada para a percepção de segurança alimentar, insegurança e fome ${ }^{2}$.

Considerando que em algumas ocasiões não é possível implementar a escala de 18 itens foi criada uma versão curta de seis itens para atuar como um substituto confiável daquele instrumento mais longo. Testes estatísticos mostraram que tal versão possui boa concordância com a medida observada pela escala completa, podendo ser usada como screening ${ }^{2}$. Porém, essa escala apresenta perda da sensibilidade e da especificidade e, em algumas ocasiões, pode não capturar o nível mais grave da insegurança alimentar, ou seja, quando ocorre fome infantil ou fome adulta ${ }^{2,3}$.

No Brasil, o instrumento baseado na escala longa norte-americana é a Escala Brasileira de Insegurança Alimentar (EBIA), que passou a ser utilizada com o intuito de contribuir à estratégia Fome Zero ${ }^{4}$. Assim, a utilização dessa escala foi proposta para auxiliar no acompanhamento e avaliação das ações desta estratégia, sendo considerada como um indicador direto para quantificar a população que poderia estar sujeita a diferentes graus de insegurança alimentar. Este instrumento caracteriza-se por avaliar a situação de insegurança alimentar familiar, incluindo tanto a percepção das pessoas, como outras questões objetivas que avaliam a disponibilidade de alimentos no domicílio ${ }^{5,6}$.

Sabe-se que escalas de insegurança alimentar fornecem informação útil para monitorar a falta de acesso aos alimentos no domicílio, em determinada população ${ }^{7}$. Considerando as duas escalas propostas ${ }^{2,4}$, o presente estudo tem por objetivo comparar a versão curta da escala de segurança alimentar, recomendada pela Food and Agriculture Organization, com a EBIA.

\section{Metodologia}

Trata-se de um estudo transversal, realizado no período de abril a outubro de 2011, na área de abrangência da Unidade de Saúde da Família (USF) de um bairro situado na periferia da cidade de Pelotas, RS.

O cálculo do tamanho amostral foi realizado por meio do Programa Epi Info, versão 6.04, sendo considerada uma estimativa de 2.922 famílias. Para o cálculo de sensibilidade e especificidade considerou-se uma prevalência de insegurança alimentar de 30\%. Dessa forma, o cálculo que exigiu maior tamanho de amostra foi para a estimativa da sensibilidade. Para uma sensibilidade de $80 \%$ e com um nível de confiança de $95 \%$ foram necessárias 60 famílias com insegurança alimentar, cuja prevalência foi estimada em 30\%, levando a uma amostra de 200 famílias. A este número foram acrescidos $10 \%$ para perdas e recusas. Tal amostra foi selecionada por meio de um sorteio sistemático a partir do cadastro existente na USF.

As entrevistas foram realizadas por acadêmicos da Faculdade de Nutrição da Universidade Federal de Pelotas (UFPel), submetidos a treinamento específico para a aplicação dos instrumentos e foram conduzidas nos domicílios dos entrevistados. A coleta de informações iniciou em maio de 2011 e teve duração de seis meses.

O instrumento utilizado abordou informações demográficas e socioeconômicas, além das questões provenientes da EBIA e da versão curta da escala de segurança alimentar.

Para a avaliação da insegurança alimentar, a pessoa responsável pela alimentação no domicílio foi questionada quanto à sua percepção sobre a situação alimentar vivida pela família. Assim, foi aplicada a EBIA que é constituída por 15 itens relativos aos três meses anteriores à entrevista. Essa escala estima prevalências de insegurança alimentar e classifica os domicílios em quatro categorias com três níveis de intensidade: segurança alimentar, insegurança alimentar leve, moderada ou grave, definidas por diferentes pontos de corte, de acordo com a composição etária do domicílio.

Para a classificação da insegurança alimentar, um escore foi calculado de acordo com o número de respostas positivas em cada item da escala (um ponto para sim e zero para não ou não sabe). Nos domicílios sem a presença de indivíduos menores de 18 anos a pontuação máxima admissível é oito, uma vez que esse é o número de questões aplicadas. Para domicílios com 
menores de 18 anos, aplicava-se o questionário completo, podendo totalizar 15 pontos. Portanto, no primeiro grupo, insegurança alimentar leve foi definida quando a soma foi de um a três pontos, moderada para aqueles domicílios que somaram de quatro a seis pontos e insegurança alimentar grave para a soma de sete a oito pontos. Já para classificar as famílias com menores de 18 anos, a pontuação para insegurança alimentar leve, moderada ou grave foi, respectivamente, para somas de um a cinco pontos, de seis a dez pontos e de onze a quinze pontos. Independente da presença ou não de menores de 18 anos, a segurança alimentar foi definida para aquelas famílias com respostas negativas em todas as questões.

Após a aplicação da EBIA, foi empregada a versão curta da escala de segurança alimentar norte-americana, que é composta por seis questões com respostas positivas e negativas, referentes aos 12 meses anteriores à entrevista. Tal questionário gera um escore que varia de zero a seis. Em quatro questões cada resposta positiva corresponde a um ponto, enquanto em uma das questões o ponto é dado na resposta negativa e na outra, a pontuação corresponde à exposição em que houve diminuição na quantidade de alimentos por falta de dinheiro. O escore foi calculado pela soma desses pontos, considerando com segurança alimentar aquelas famílias que atingiram entre zero e um ponto; insegurança alimentar sem fome para aquelas que somaram entre dois e quatro pontos e com fome quando o escore atingiu cinco ou seis pontos.

A entrada de dados foi realizada com dupla digitação, sendo utilizado o programa EpiData, versão 3.1. Posterior análise foi realizada no programa Stata, versão 12.0 (Stata Corp., College Station, Estados Unidos).

Para comparar as duas escalas, testaram-se diferentes modelos de classificação da situação de insegurança alimentar. No primeiro modelo, calculou-se a prevalência de insegurança alimentar com os pontos de corte tradicionais das duas escalas, supracitados nesta seção. A seguir, modificou-se o ponto de corte da EBIA, considerando como inseguros aqueles domicílios com duas ou mais respostas positivas. Tal mudança teve o objetivo de aproximar o ponto de corte dessa escala àquele da versão curta. No terceiro modelo alterou-se o ponto de corte da versão curta, considerando como inseguros aqueles domicílios que tiveram uma resposta positiva. $\mathrm{O}$ intuito de tal mudança foi aproximar o ponto de corte dessa escala àquele da EBIA.
Os índices de sensibilidade, especificidade, valores preditivos positivo e negativo e acurácia foram calculados para a versão curta em relação à EBIA, considerada o padrão-ouro. Também foram estimadas prevalências a partir desses índices com diferentes pontos de corte para ambas as escalas.

Por fim, para avaliar a concordância entre as duas escalas, aplicou-se o teste Kappa. Considerou-se um Kappa de 0,0 a 0,3 como concordância fraca, de 0,31 a 0,7 como concordância moderada, 0,71 a 0,99 como concordância satisfatória e 1,0 para uma concordância perfeita entre as duas escalas. Para esta análise também foram utilizados diferentes pontos de corte, com o propósito de detectar maior concordância entre as escalas ${ }^{8}$.

Para todas as análises já mencionadas considerou-se o total de domicílios, bem como a estratificação dos mesmos segundo a presença ou não de menores de 18 anos.

O estudo foi aprovado pelo Comitê de Ética em Pesquisa da Faculdade de Medicina da Universidade Federal de Pelotas. Todos os entrevistados assinaram um termo de consentimento livre e esclarecido.

\section{Resultados}

Foram visitadas 230 famílias, sendo que aproximadamente $57 \%$ dos chefes dos domicílios entrevistados eram do sexo masculino, dos quais pouco menos de $20 \%$ tinha nove ou mais anos de estudo e $73 \%$ apresentavam idade inferior a 60 anos.

Em relação aos resultados sobre a situação de segurança alimentar das famílias estudadas, observou-se que, segundo a EBIA, mais da metade convivia com algum nível de insegurança, sendo que destes assim classificados, a maioria vivia com insegurança alimentar leve, isto é, sem a presença de fome. $\mathrm{Na}$ análise estratificada pela composição etária do domicílio, observou-se que naqueles onde não havia menores de 18 anos, pouco mais da metade apresentou algum nível de insegurança alimentar, sem a presença da forma grave. Já nos domicílios com menores de 18 anos, a insegurança alimentar atingiu 2/3 dos entrevistados (Tabela 1).

Ainda na Tabela 1 é possível observar que, segundo a versão curta, apenas $1 / 4$ da amostra vivia em situação de insegurança alimentar. Ademais, na análise estratificada, constatou-se que nos domicílios sem menores de 18 anos essa pre- 
Tabela 1. Prevalência de insegurança alimentar de acordo com as duas escalas (EBIA e versão curta). Pelotas, Brasil, 2011.

\begin{tabular}{lrcr}
\hline \multicolumn{1}{c}{ Variáveis } & $\begin{array}{c}\text { Total } \\
\mathbf{n}(\%)\end{array}$ & $\begin{array}{c}\text { Domicílios sem }<\mathbf{1 8} \text { anos } \\
\mathbf{n}(\mathbf{\%})\end{array}$ & $\begin{array}{r}\text { Domicílios com }<\mathbf{1 8} \text { anos } \\
\mathbf{n}(\mathbf{\%})\end{array}$ \\
\hline EBIA $^{\text {a }}$ & & & $54(35,8)$ \\
$\quad$ Segurança alimentar & $91(39,6)$ & $37(46,8)$ & $75(49,7)$ \\
IA leve & $112(48,7)$ & $37(46,8)$ & $15(9,9)$ \\
IA moderada & $20(8,7)$ & $5(6,4)$ & $7(4,6)$ \\
IA grave & $7(3,0)$ & $0(0,0)$ & $76(50,3)$ \\
EBIA & & & $53(35,1)$ \\
Segurança alimentar & $128(55,7)$ & $52(65,8)$ & $22(14,6)$ \\
IA sem fome & $75(32,6)$ & $22(27,9)$ & $111(73,5)$ \\
IA com fome & $27(11,7)$ & $5(6,3)$ & $25(16,6)$ \\
Versão curta & & & $15(9,9)$ \\
Segurança alimentar & $174(75,6)$ & $63(79,8)$ & $97(64,2)$ \\
IA sem fome & $35(15,3)$ & $10(12,7)$ & $39(25,8)$ \\
IA com fome & $21(9,1)$ & $6(7,5)$ & $15(9,9)$ \\
Versão curta & & & \\
Segurança alimentar & $151(65,7)$ & $54(68,4)$ & $(24,1)$ \\
IA sem fome & $58(25,2)$ & $6(7,5)$ & \\
IA com fome & $21(9,1)$ & &
\end{tabular}

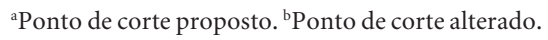

Tabela 2. Teste de concordância entre a EBIA e a versão curta norte-americana. Pelotas, Brasil, 2011.

\begin{tabular}{lcc}
\hline \multicolumn{1}{c}{ Estatística Kappa } & IA total & IA com fome \\
\hline $\begin{array}{l}\text { Todos os domicílios } \\
\text { Modelo } 1^{\mathrm{a}}\end{array}$ & 0,33 & 0,58 \\
Modelo 2 $^{\mathrm{b}}$ & 0,48 & 0,58 \\
$\quad$ Modelo $3^{\mathrm{c}}$ & 0,40 & 0,58 \\
Domicílios sem < 18 anos & & \\
$\quad$ Modelo $1^{\mathrm{a}}$ & 0,32 & 0,71 \\
Modelo 2 & 0,47 & 0,71 \\
Modelo $3^{\mathrm{c}}$ & 0,38 & 0,71 \\
Domicílios com < 18 anos & & \\
Modelo $1^{\mathrm{a}}$ & 0,33 & 0,54 \\
Modelo 2 & 0,48 & 0,54 \\
Modelo $3^{\mathrm{c}}$ & 0,40 & 0,54 \\
\hline
\end{tabular}

${ }^{a}$ EBIA e versão curta com ponto de corte proposto. ${ }^{\mathrm{b}}$ EBIA com ponto de corte alterado e versão curta com ponto de corte proposto. ${ }^{c}$ Versão curta com ponto de corte alterado e EBIA com ponto de corte proposto.

valência foi de $20 \%$, sendo mais elevada nos domicílios onde havia crianças ou adolescentes. A mudança do ponto de corte dessa escala, ao contrário do que é observado para a EBIA, aumentou a prevalência de insegurança alimentar em dez pontos percentuais (de $24,4 \%$ para $34,3 \%$ ), independente da presença ou não de menores de 18 anos.
A comparação entre as duas escalas mostra concordância moderada tanto para analisar insegurança alimentar como um todo, quanto para avaliar uma possível situação de fome, mesmo alterando o ponto de corte das duas escalas (Tabela 2). Por outro lado, concordância satisfatória foi observada somente quando analisada a situação de fome em domicílios sem menores de 18 anos.

Na Tabela 3 verifica-se a análise dos parâmetros de validade da versão curta, constatando-se que esta escala apresenta baixa sensibilidade e alta especificidade em relação à EBIA, independente do ponto de corte observado. Em contrapartida, constata-se que para avaliar a situação de fome, tal escala se apresenta um bom parâmetro, visto que para o ponto de corte proposto, isto é, igual ou maior a cinco, ela apresentou alta sensibilidade e especificidade.

Ademais, para o ponto de corte proposto pela versão curta, a proporção de pessoas com resultado positivo que, de fato, apresentavam insegurança alimentar pela EBIA (valor preditivo positivo) foi de quase $100 \%$. Por outro lado, entre aqueles que não tinham insegurança alimentar pela EBIA, menos de 50\% foram assim detectados pela versão curta (valor preditivo negativo). Por fim, com relação à acurácia, isto é, a probabilidade de o teste estar de acordo com o pa- 
Tabela 3. Sensibilidade e especificidade da versão curta da escala de segurança alimentar domiciliar norte-americana. Pelotas, Brasil, 2011.

\begin{tabular}{lcc}
\hline \multicolumn{1}{c}{ Pontos de corte } & Sensibilidade $($ IC 95\%) & Especificidade $($ IC 95\%) \\
\hline Insegurança alimentar & & \\
$\geq 1$ & $51,8(43,2-60,3)$ & $92,3(84,8-96,9)$ \\
$\geq 2$ & $39,6(31,4-48,2)$ & $98,9(94,0-100)$ \\
$\geq 3$ & $24,5(17,6-32,5)$ & $100(96,0-100)$ \\
$\geq 4$ & $21,6(15,1-29,4)$ & $100(96,0-100)$ \\
$\geq 5$ & $15,1(9,6-22,2)$ & $100(96,0-100)$ \\
6 & $7,19(3,5-12,8)$ & $100(96,0-100)$ \\
Fome & & \\
$\geq 1$ & $90,9(58,7-99,8)$ & $68,5(61,9-74,6)$ \\
$\geq 2$ & $90,9(58,7-99,8)$ & $79,0(73,0-84,2)$ \\
$\geq 3$ & $90,9(58,7-99,8)$ & $89,0(84,1-92,9)$ \\
$\geq 4$ & $81,8(48,2-97,1)$ & $90,4(85,7-94,0)$ \\
$\geq 5^{* *}$ & $81,8(48,2-97,1)$ & $94,5(90,6-97,1)$ \\
6 & $72,7(39,0-94,0)$ & $99,1(96,7-99,9)$ \\
\hline
\end{tabular}

* Ponto de corte para insegurança alimentar da versão curta. ${ }^{* *}$ Ponto de corte para fome da versão curta.

drão-ouro, o valor encontrado foi de $63 \%$ para todas as famílias avaliadas. Mesmo alterando o ponto de corte da escala norte-americana, igualando-o àquele proposto pela EBIA, não foi suficiente para alterar significativamente os resultados dos parâmetros mencionados (dados não mostrados).

Ao estratificarem-se os domicílios conforme composição etária observa-se que os valores referentes à sensibilidade, especificidade, valores preditivos positivo e negativo e acurácia apresentaram resultados semelhantes àqueles referentes ao total de domicílios. Além disso, para a situação de fome, a versão curta também apresentou melhor capacidade de acertar os resultados em relação à EBIA nesta análise. Por fim, cabe salientar um aumento considerável na sensibilidade quando são considerados somente os domicílios sem crianças ou adolescentes (Tabela 4).

\section{Discussão}

O principal resultado deste estudo se refere à diferença na medida de insegurança alimentar de acordo com as duas escalas, que se manteve mesmo com as mudanças nos pontos de corte de ambas. Segundo a EBIA, mais da metade da amostra apresentou algum nível de insegurança alimentar; já conforme a versão curta de seis questões, apenas 1/4 das famílias vivia nesta condição. Essa diferença pode ser explicada pelo número de falsos-negativos da versão curta, resul- tando em baixa sensibilidade dessa escala em relação à EBIA. Dessa forma, aqueles identificados como inseguros pela versão curta têm maior probabilidade de serem, de fato, domicílios com insegurança alimentar.

Outro aspecto fundamental deste estudo é o fato de ser o primeiro a comparar a EBIA, escala que foi validada para a população brasileira entre os anos de 2003 e 2004, com uma versão curta, recomendada pelo FAO e que classifica as famílias com ou sem fome. A utilização de uma escala mais curta comparada à versão atual validada para a população brasileira pode ser positiva por ser de fácil aplicação e de baixo custo, facilitando o desenvolvimento de estudos cujo propósito seja verificar e/ou monitorar a situação alimentar de famílias brasileiras.

Limitações devem ser consideradas, como o fato de que as duas escalas refletem uma medida relativa da situação experimentada por todos os indivíduos vivendo em um mesmo domicílio, não sendo uma medida individual de insegurança alimentar. No entanto, apesar dessa limitação, sabese que quando um morador do domicílio experimenta essa situação, consequentemente todos os moradores podem estar afetados ${ }^{2}$. Outra limitação diz respeito ao diferente tempo recordatório das duas escalas. Enquanto a EBIA se refere aos três meses anteriores à entrevista, a versão curta trata dos doze meses precedentes à aplicação do questionário.

A EBIA tem sido amplamente utilizada no Brasil, sendo relevante no combate à desigualda- 
Tabela 4. Sensibilidade, especificidade, valores preditivos positivos e acurácia da versão curta da escala de segurança alimentar domiciliar norte-americana, estratificados por composição etária do domicílio. Pelotas, Brasil, 2011.

\begin{tabular}{lcc}
\hline \multicolumn{1}{c}{ Diagnóstico } & Domicílios sem $<\mathbf{1 8}$ anos & Domicílios com $<\mathbf{1 8}$ anos \\
\hline Determinação da IA total & & \\
Sensibilidade & $35,7 \%$ & $41,2 \%$ \\
Especificidade & $97,3 \%$ & $100 \%$ \\
Valor Preditivo Positivo & $93,8 \%$ & $100 \%$ \\
Valor Preditivo Negativo & $57,1 \%$ & $48,7 \%$ \\
Acurácia & $64,6 \%$ & $62,3 \%$ \\
Determinação de IA com fome & & \\
Sensibilidade & $80,0 \%$ & $50,0 \%$ \\
Especificidade & $97,3 \%$ & $96,9 \%$ \\
Valor Preditivo Positivo & $66,7 \%$ & $73,3 \%$ \\
Valor Preditivo Negativo & $98,6 \%$ & $91,9 \%$ \\
Acurácia & $96,2 \%$ & $90,1 \%$ \\
Determinação da IA total & & \\
Sensibilidade & & $52,6 \%$ \\
Especificidade & $50,0 \%$ & $94,4 \%$ \\
Valor Preditivo Positivo & $89,2 \%$ & $94,4 \%$ \\
Valor Preditivo Negativo & $84,0 \%$ & $52,6 \%$ \\
Acurácia & $61,1 \%$ & $67,6 \%$ \\
\hline
\end{tabular}

*Versão curta com ponto de corte alterado.

de social do país, principalmente no que diz respeito ao acesso aos alimentos e no monitoramento da insegurança alimentar ${ }^{6}$. Além disso, outros determinantes aparecem atrelados a essas situações, como cor da pele, escolaridade e gênero da pessoa de referência do domicílio ${ }^{9,10}$, evidenciando a importância da medida da insegurança alimentar domiciliar, possibilitando investigar indivíduos com potencial para ingressar em programas sociais e de transferência de renda.

No que se refere à prevalência de insegurança alimentar a partir de dados da última Pesquisa Nacional de Demografia e Saúde (PNDS), constatou-se que a proporção de famílias com insegurança alimentar no Rio Grande do Sul foi de $37,5 \%{ }^{11}$. Em contrapartida, no presente estudo a proporção de famílias com insegurança alimentar segundo a EBIA foi quase o dobro quando comparada à média estadual.

Ao analisar-se a prevalência de insegurança alimentar de acordo com a versão curta, observou-se que cerca de 25\% das famílias analisadas apresentaram tal problema. Em outro estudo que utilizou a versão curta norte-americana como método para detectar a prevalência de insegurança alimentar, realizado na cidade de Pelotas (RS), observou-se que $11 \%$ das famílias conviviam com esta situação ${ }^{12}$. É importante salientar que, diferentemente dos estudos menciona- $\operatorname{dos}^{11,12}$, o presente trabalho foi realizado com uma população específica de uma localidade de baixa renda, não sendo de base populacional.

Quando proposta, em 1999, a versão curta foi comparada à escala de 18 itens, também recomendada pelo USDA, e apresentou boa concordância geral, identificando como inseguros 97,7\% dos domicílios que tinham insegurança alimentar segundo a escala completa, apresentando sensibilidade e especificidade de mais de $90 \%{ }^{3}$. Contudo, no presente trabalho, observou-se que a versão curta só apresentou concordância satisfatória com o padrão-ouro para avaliar a situação de fome em domicílios sem menores de 18 anos. Ademais, a sensibilidade máxima obtida na análise foi apenas para detectar insegurança alimentar grave, ou seja, quando já há presença de fome no domicílio. Sabe-se que para uma escala ser considerada um bom método de rastreamento, necessita ter alta sensibilidade, isto é, alta capacidade de identificar como doente quem realmente possui a doença em questão ${ }^{13}$. Assim, a partir dos resultados supracitados, percebe-se que a versão curta, quando comparada à EBIA, não é um bom método de rastreamento da insegurança alimentar como um todo para famílias brasileiras.

Visto que a insegurança alimentar pode acarretar diversos agravos como fome, desnutrição, carências específicas, excesso de peso, doenças 
geradas pela alimentação inadequada e consumo de produtos prejudiciais à saúde ${ }^{5,14}$, é oportuno e relevante identificar indicadores que possam verifi-car tal situação. O Plano Nacional de Segurança Alimentar e Nutricional - 2012/2015 evidencia que é necessária a construção de metodologias que monitorem a realização progressiva do Direito Humano a Alimentação Adequada (DHAA $)^{9}$, envolvendo um espectro amplo de indicadores e diretrizes. Alguns destes indicadores, bem como o processo de coleta de dados para construção dos mesmos, podem ser tão ou mais complexos que a EBIA ${ }^{15}$. Sendo assim, a criação de um instrumento menor de medida da insegurança alimentar, baseado na escala brasileira, seria de grande importância para situações que necessitem medir o nível domiciliar de insegurança alimentar, onde não haja tempo e recursos suficientes para aplicação da escala completa ou onde se necessitem medir inúmeros indicadores para monitorar a realização do DHAA.

É inequívoco que muitos municípios brasileiros enfrentam escassez de recursos financeiros e necessitem de estratégias de baixo custo para a promoção da inclusão social e diminuição da desigualdade, detectando pessoas elegíveis para diversos programas sociais. Portanto, uma ver- são mais sucinta da escala de insegurança alimentar, que mantivesse os indicadores essenciais de insegurança alimentar e apresentasse bons índices de concordância geral quando comparada à EBIA, poderia ir ao encontro dessa necessidade, mesmo sabendo que programas sociais, por si só, não garantam índices satisfatórios de segurança alimentar, questão associada a um quadro de pobreza mais amplo ${ }^{16,17}$.

\section{Conclusão}

A versão curta proposta pelo USDA apresenta baixa concordância quando comparada à EBIA, método validado para a população brasileira. Dessa forma, urge a criação de um instrumento menor, de fácil e rápida aplicação e de baixo custo, com base na EBIA, funcionando como uma espécie de screening para identificação de famílias vulneráveis. O referido instrumento de avaliação da insegurança alimentar deverá ser capaz de rastrear com facilidade famílias que vivenciam tal situação, tornando-se assim útil para a decisão da sua inclusão ou da sua manutenção como beneficiários diretos de programas e políticas públicas direcionadas.

\section{Colaboradores}

LP Santos foi responsável pela revisão de literatura, análise dos dados, confecção das tabelas, interpretação e redação do artigo final. MG Costa, JV Santos, IL Lindemann e DP Gigante foram responsáveis pela interpretação dos dados, redação e revisão do artigo final. 


\section{Referências}

1. Brasil. Lei no 11.346 , de 15 de setembro de 2006. Cria o Sistema Nacional de Segurança Alimentar e Nutricional - SISAN com vistas em assegurar o direito humano à alimentação adequada e dá outras providências. Diário Oficial da União 2006; 18 set.

2. Bickel GW, Nord M, Price C, Hamilton W, Cook J. Measuring food security in the United States. Guide to measuring household food security. Revised 2000. Alexandria: United States Department of Agriculture; 2000.

3. Blumberg SJ, Bialostosky K, Hamilton WL, Briefel RR. The effectiveness of a short form of the Household Food Security Scale. Am J Public Health 1999; 89(8):1231-1234.

4. Perez-Escamilla R, Segall-Correa AM, Maranha LK, Sampaio MFA, Marin-Leon L, Panigassi G. An adapted version of the U.S. Department of Agriculture Food Insecurity module is a valid tool for assessing household food insecurity in Campinas, Brazil. J Nutr 2004; 134(8):1923-1928.

5. Segall-Corrêa AM. Insegurança alimentar medida a partir da percepção das pessoas. Estud Av 2007; 21(60):143-154.

6. Segall-Corrêa AM, Marin-Leon L. A Segurança Alimentar no Brasil: Proposição e Usos da Escala Brasileira de Medida da Insegurança Alimentar (EBIA) de 2003 a 2009. Segurança Alimentar e Nutricional, Campinas 2009; 16(2):1-19.

7. Knueppel D, Demment M, Kaiser L. Validation of household food insecurity access scale in rural Tanzania. Public Health Nutrition 2009; 13(3):360-367.

8. Fletcher R, Fletcher S. Epidemiologia Clínica. $4^{\text {a }}$ Edição. São Paulo: ArtMed; 2006.

9. Brasil. Ministério do Desenvolvimento Social. Plano Nacional de Segurança Alimentar e Nutricional: 2012/2015. Brasília: Câmara Interministerial de Segurança Alimentar e Nutricional; 2011.

10. Instituto Brasileiro de Geografia e Estatística (IBGE). Pesquisa Nacional de Amostra de Domicílios. Segurança Alimentar 2004-2009. Rio de Janeiro: IBGE; 2010.
11. Brasil. Ministério da Saúde (MS). Relatório Final da Pesquisa Nacional de Demografia e Saúde. Brasília: Pesquisa Nacional de Demografia e Saúde da Criança e da Mulher; [página na Internet] 2006. [acessado 2013 out 26]. Disponível em: http://www.saude. gov.br/pnds2006

12. Santos JV, Gigante DP, Domingues MR. Prevalência de insegurança alimentar em Pelotas, Rio Grande do Sul, Brasil, e estado nutricional de indivíduos que vivem nessa condição. Cad Saude Publica 2010; 26(1):41-49.

13. Gordis L. Epidemiology. $4^{a}$ Edição. Baltimore: Elsevier; 2008.

14. Salles-Costa R. Avaliação alimentar em Duque de Caxias (RJ). Estud. Avan 2007; 21(60):135-142.

15. Kepple AW, Segall-Corrêa AM. Conceituando e medindo segurança alimentar e nutricional. Cien Saude Colet 2011; 16(1):187-199.

16. Brasil. Instituto Brasileiro de Análises Sociais e Econômicas. Repercussões do Programa Bolsa Família na Segurança Alimentar e Nutricional das Famílias Beneficiadas. Rio de Janeiro: IBASE; 2008.

17. Ramos CI, Cuervo MRM. Programa Bolsa Família: a interface entre a atuação profissional e o direito humano a alimentação adequada. Cien Saude Colet 2012; 17(8):2159-2168.

Artigo apresentado em 06/11/2012

Aprovado em 11/12/2012

Versão final apresentada em 26/12/2012 